\title{
Simulation and estimation of parameters in reconfigurable multipoint forming processes of plates in the creep mode
}

\author{
Konstantin Bormotin ${ }^{1,}$, Sergey Belykh ${ }^{1}$, and Vin Aung ${ }^{1}$ \\ ${ }^{1}$ Komsomolsk-na-Amure State Technical University, 681013, Komsomolsk-on-Amur, Russia
}

\begin{abstract}
Mathematical models and numerical methods for the optimal solution of inverse forming problems monolithic panels with contact stamp devices have been developed. Using the minimization criterion for damage in functionals of inverse problems, the optimal laws of deformation in creep are found. The algorithms implemented in MSC.Marc allow to model and evaluate the parameters of the technological processes for the manufacture of parts, in particular reconfigurable multi-point form-creation.
\end{abstract}

\section{Introduction}

Lately in the aircraft industry, large monolithic and monolithic prefabricated panels are used more and more as parts, which allow increasing strength and stiffness of structures with simultaneous reduction of masses and improvement of aerodynamic performances. Monolithic panels utilize reinforced membranes fabricated in conjunction with the stiffening ribs of one plate or sheet [1]. Setting the aerodynamic shape of such monolithic panels is performed by shaping processes undertaken in plastic deformation modes, both under normal and increased temperatures.

The development of metal forming processes is basically either through the creation of highly efficient technologies based on optimization of regimes, or the development of new technological processes. Establishment of the most effective technological process of deformation leads to the problem of choosing optimal technological parameters. In any case, the most important criteria necessary for assessing the effectiveness of technological processes and their optimal management of technology parameters are determined for the solution, the objective function characterizing the process and being a function of various factors is introduced. In a number of works, a set of problems was solved to optimize the parameters of the force loading and deformation of thin sheet billets under pulsed methods of shaping, in particular, the multiparametric problem of optimal shape formation was posed and solved [2].

The process of deformation in the creep regime allows for economically and efficiently forming large parts. The use of shaping such a regime avoids many manual operations by mechanical molding.

The deformation-strength properties of the creep of structural aluminum alloys depend significantly on the state of supply, the thickness of the slab, and the direction of cutting the blanks from the plate (slab) for making samples and the sign of the applied load during the tests [3]. It is experimentally shown in works [4-10] that forming in the creep regime and at stresses not exceeding the elastic strength of material, provides for higher residual strength resource and less damage of components compared to the method of conventional cold forging. The results confirm the prospects of creep using in the manufacturing processes of critical structures parts. This is especially important for modern civil and transport aircraft, since the airworthiness standards are continually being increased and are currently based on residual strength estimates and damage tolerance of the basic design elements [11, 12].

Such processes, in addition, will allow controlling the level of material damage, coordinated with technological limitations, due to the optimal choice of the strain path in time.

Development of technical decisions in ways of shaping does not guarantee high accuracy of final geometrical shape of the part. For example, lately shaping of products from sheets and panels is considered as a promising solution, using configurable core punch (die), which allows changing the shape of workpiece. Accuracy of the part shape obtained by pressure processing technologies of materials under specified process parameters depends on accuracy of calculated and manufactured tooling shape (stamps, punch, rod configurable punch) that determines proactive panel shape. Proactive panel shape shall provide specified residual curvature of the panel after its release from the power tooling. In this regard, inverse problem rises: to determine the shape of tooling, which creates such proactive curvature of the panel that provides specified residual shape of the panel after unloading. In addition, in order to minimize damage to parts and deviations from specified shape under existing limitations in production, formulation of process parameters optimal control problems and development of methods of their solution are required.

\footnotetext{
* Corresponding author: cvmi@knastu.ru
} 
It's shown in works $[13,14]$ that panel formation in the creep regime is limited by deformation and shows a large value of material spring-back. To account the spring-back and to obtain the required curvature of the panel it is proposed to use multi-point forming (MPF) in the creep regime [15].

The transition of forming matrixes and punches from rigid to flexible structures, enabling changes to their shape under the required geometry have opened broad possibilities for the application of reconfigurable tooling system in the variety of technological processes. The forming surface of the punch as well as the matrix formed by two coaxially arranged rods systems, each placed in an individual position by the numerical control, allows for the adaptation of the tooling system for manufacturing parts of various configurations. The mechanisms of rods positioning can be mechanical, hydraulic or combined. There are also differences in the configuration of the rods, their tips and the quality of formed surface of the punch (the matrix).

Most of the research exploring the possibility of using the reconfigurable rod devices is aimed to develop the technology of work-piece manufacturing from thin sheet material. In works [16-19] design features of rod systems are considered and the main approaches to definition of the loads acting on each rod element are proposed. Results of searches for the most rational form of rod element are also presented.

Results of complex research for the prediction of defect occurrence of multi-point forming of sheet material by utilizing finite-element analyses (FEM) are shown in works [20-22]. The obtained data allowed for the optimization of the control system for the punch and matrix rods and gave the opportunity to manufacture the parts using double curvature from the sheet material with account of the effects of material spring-back.

\section{Formulation and method for solving inverse optimal forming problems}

Let $V \subset R^{3}$ be a bounded domain with a sufficiently regular boundary $S$. The contact surface of rigid bodies (stamps) with deformable body is designated through $S_{c}$ $\left(S_{c} \subset S\right)$. Denote by $u=\left(u_{1}, u_{2}, u_{3}\right), \quad \tilde{u}=\left(\tilde{u}_{1}, \tilde{u}_{2}, \tilde{u}_{3}\right)$, $\bar{u}=\left(\bar{u}_{1}, \bar{u}_{2}, \bar{u}_{3}\right)$ - the vectors of current, residual displacements of deformable body and the vectors displacements contact surface of rigid bodies; $u, \tilde{u} \in\left[W_{2}^{1}(Q)\right]^{3}, \quad Q=V \times\{0 \leq t \leq T\}, \quad \bar{u} \in\left[W_{2}^{1}\left(Q_{c}\right)\right]^{3}$, $Q_{c}=S_{c} \times\{0 \leq t \leq T\}$. The symbol $(\cdot, \cdot)_{S}$ denotes the inner product in $L_{2}(S):(u, v)_{S}=\int_{S} \sum_{i=1}^{3} u_{i} v_{i} d S$. The corresponding norm is given by $\|u\|_{S}=\sqrt{(u, u)_{S}}=\left(\int_{S} \sum_{i=1}^{3} u_{i}^{2} d S\right)^{1 / 2}$.

The inverse problem of a kinematic forming by contact rigid stamps can be represented in the form of a quasi-static variational principle with the functional [23]:

$$
\begin{gathered}
J(\dot{\bar{u}}, \dot{u}, \dot{\tilde{u}})=\frac{1}{2 \varepsilon_{1}}\left\|\dot{\bar{u}}-\dot{\bar{u}}^{*}\right\|_{S_{c}}{ }^{2}+W_{c}+a(\dot{u}, \dot{u})+ \\
+a(\dot{\tilde{u}}, \dot{\tilde{u}})+\frac{1}{2 \varepsilon_{2}}\left\|\dot{\tilde{u}}-\dot{\tilde{u}}^{*}\right\|_{S}{ }^{2}, \varepsilon_{1}>0, \varepsilon_{1} \rightarrow 0, \\
\varepsilon_{2}>0, \varepsilon_{2} \rightarrow 0,
\end{gathered}
$$

where $\dot{\tilde{u}}^{*}, \dot{\bar{u}}^{*}$ is a given residual displacement rate and current contact displacement rate in moment time $t$; $t \in[0, T]$ - time deformation under load; $W_{c}$ - the quasistatic contact potentials received by imposing of contact conditions on the equations of bodies motion by method of multipliers of Lagrange or by method of penal functions [24] and differentiation on $t$; potential form are given by:

$$
\begin{gathered}
a(\dot{u}, \dot{v})=\int_{V} \frac{\partial W\left(\dot{\varepsilon}_{i j}(\dot{u})\right)}{\partial \dot{\varepsilon}_{i j}(\dot{u})} \dot{\varepsilon}_{i j}(\dot{v}) d V, \\
\left.a(\dot{\tilde{u}}, \dot{\tilde{v}})=\int_{V}\left(\frac{\partial W\left(\dot{\tilde{\varepsilon}}_{i j}(\dot{\tilde{u}})\right)}{\partial \dot{\tilde{\varepsilon}}_{i j}(\dot{\tilde{u}})}\right) \dot{\tilde{\varepsilon}}_{i j} \dot{\tilde{v}}\right) d V, \\
W\left(\dot{\varepsilon}_{i j}\right)=\frac{1}{2} c_{i j k l} \dot{\varepsilon}_{i j} \dot{\varepsilon}_{k l}-c_{i j k l} \dot{\varepsilon}_{i j} \dot{\varepsilon}_{k l}^{c},
\end{gathered}
$$

$W\left(\dot{\tilde{\varepsilon}}_{i j}\right)=\frac{1}{2} c_{i j k l} \dot{\tilde{\varepsilon}}_{i j} \dot{\tilde{\varepsilon}}_{k l}-c_{i j k l} \dot{\tilde{\varepsilon}}_{i j} \dot{\varepsilon}_{k l}^{c}, c_{i j k l}$ are the components of the symmetric elastic constant tensor; $\dot{\varepsilon}_{k l}^{c}$ are the creep strain rates; $i, j, k, l=1,2,3 ; \dot{\varepsilon}_{i j}=\frac{1}{2}\left(\dot{u}_{i, j}+\dot{u}_{j, i}\right)$, $\dot{\tilde{\varepsilon}}_{i j}=\frac{1}{2}\left(\dot{\tilde{u}}_{i, j}+\dot{\tilde{u}}_{j, i}\right)$.

By consideration of the inverse problem with functional in the form of (1) it is supposed that in the considered time $t$ there is a contact of rigid stamp with deformable body, otherwise these contact potentials shouldn't enter functional.

The problem of optimal deformation is formulated as follows: it is required to find a way to deform an element of the medium for a given time $T$, so that at the time $t=T$, the specified creep strains $\dot{\varepsilon}_{k l}^{c^{*}}$ are obtained and the damage parameter $\Omega$ is minimal.

Minimizing the dissipation power for each time point will give the minimum value of the dissipated work $A=\int_{0}^{T} \sigma_{i j} \dot{\varepsilon}_{i j}^{c} d t$ and accordingly the damage parameter $[25,26]$.

Using the weighting coefficients method, the problem of optimal deformation by the functional $[25$, 26]

$$
\begin{aligned}
& J(\dot{\bar{u}}, \dot{u}, \dot{\tilde{u}})=\frac{1}{2 \varepsilon_{1}}\left\|\dot{\bar{u}}-\dot{\bar{u}}^{*}\right\|_{S_{c}}{ }^{2}+W_{c}+a(\dot{u}, \dot{u})+ \\
& +c_{1}\left\{a(\dot{\tilde{u}}, \dot{\tilde{u}})+\frac{1}{2 \varepsilon_{2}}\left\|\dot{\tilde{u}}-\dot{\tilde{u}}^{*}\right\|_{S}{ }^{2}\right\}+c_{2} \int_{V} \sigma_{i j} \dot{\varepsilon}_{i j}^{c} d V,
\end{aligned}
$$

where $c_{1}>0, c_{2}>0$ - weighting coefficients. 
Thus, in the case of small deflections, the optimal deformation is linear $u_{z}=\frac{t}{T} u_{z}{ }^{*}$, in the case of large deflections with the assumptions made above, optimal deformation is carried out by the nonlinear law $u_{z}=\sqrt{\frac{t}{T}} u_{z}^{*}[25]$.

In the inverse problem of uniaxial stretching of a rod in the creep theory with minimal damage, the dependence of the rational loading path on the weight coefficients and time in the following form [26]:

$$
p=\left[\frac{E B\left(1+c_{1}\right)}{c_{2}}+\left(p^{*(1-n)}-\frac{E B\left(1+c_{1}\right)}{c_{2}}\right) e^{c_{2}(1-n) t}\right]^{\frac{1}{1-n}},
$$

where $p=p(t)$ - tensile forces applied to the $\operatorname{rod}, E, B$, $n$ - material constants и $p^{*}$ - initial load. A similar expression is constructed for displacements.

It is proposed in [27] to determine the optimal loading path by formulas

$$
\begin{gathered}
u_{z}\left(t, x_{1}, x_{2}\right)=\frac{f(t)}{f(T)} u_{z}^{*}\left(x_{1}, x_{2}\right), \\
f(t)=\left[\frac{E B\left(1+c_{1}\right)}{c_{2}}+\left(\left[\frac{1}{B}\right]^{\frac{1}{n}}-\frac{E B\left(1+c_{1}\right)}{c_{2}}\right) e^{c_{2}(1-n) t}\right]^{\frac{n}{1-n}},
\end{gathered}
$$

where the values of the weight coefficients are found by the method of local variations.

Taking into account discretization of the variation (1), (2), the finite element equations for the solution of a contact problem are formed [24, 28]

$$
{ }^{t+\Delta t} \mathbf{K}^{(i-1)} \Delta \mathbf{U}^{(i)}={ }^{t+\Delta t} \mathbf{R}^{(i-1)},{ }^{t+\Delta t} \tilde{\mathbf{K}}^{(i-1)} \Delta \hat{\mathbf{U}}^{(i)}={ }^{t+\Delta t} \hat{\mathbf{R}}^{(i-1)}
$$

where ${ }^{t+\Delta t} \mathbf{K}^{(i-1)},{ }^{t+\Delta t} \tilde{\mathbf{K}}^{(i-1)}$ - are the tangent stiffness matrices (in matrices ${ }^{t+\Delta t} \mathbf{K}^{(i-1)}$ are already included the additional elements which are formed from contact restrictions), ${ }^{t+\Delta t} \mathbf{R}^{(i-1)},{ }^{t+\Delta t} \hat{\mathbf{R}}^{(i-1)}$ - the vector of internal and external forces. The top indexes $t+\Delta t$ of quantity indicate time for which it is calculated. The top indexes $(i-1)$ indicate number of iteration at correction of the solution by Newton-Rafson's method. The solution of the following step is found on a formula ${ }^{t+\Delta t} \mathbf{U}={ }^{t} \mathbf{U}+\Delta \mathbf{U}$.

As a result of the solution of the first problem (2) of the given movements we receive the deformed model with distribution of stress and strain. The second problem on the basis of data on initial stress and strain determines unloading movements. After that it is possible to find residual nodal movements $\tilde{\mathbf{U}}=\mathbf{U}+\hat{\mathbf{U}}$.

Several procedures have been developed to treat contact problems including the use of Perturbed or Augmented Lagrangian methods, penalty methods, [24, 28] and direct constraints. MSC.Marc divides contact problems into two domains [29]: the first is when a deformable body makes contact with a rigid surface and the second is when a deformable body makes contact with another deformable body or itself. A target node on the deformable body has no constraint while contact does not occur. Once contact is detected, the degrees of freedom are transformed to a local system and a constraint is imposed such that $\Delta u_{\text {normal }}=\mathbf{v} \cdot n$, where $\mathbf{v}$ is the prescribed velocity of the rigid surface.

The iterative method for solving the inverse forming problem in displacement of a contact surface is offered

$$
\bar{u}_{i}^{k+1}=\bar{u}_{i}^{k}+\alpha^{k}\left(\tilde{u}_{i}^{*}-\tilde{u}_{i}^{k}\right)+\beta^{k}\left(\bar{u}_{i}^{k}-\tilde{u}_{i}^{k}\right)
$$

where $0<\alpha^{k}<2, \beta^{k} \rightarrow 0$ at $k \rightarrow \infty, i=1,2,3$.

\section{Numerical results for the inverse problems}

The problem of movement's determination of the cores of reconfigurable tool creating such anticipatory curvature of the panel which provides the set residual form of the panel after unloading is considered. For modeling the process of forming the work-piece from plate with a thickness of $45 \mathrm{~mm}$, finite-element model (FEM) in the CAE system MSC.Marc has been developed (Fig.1). The perfectly rigid body with a tip diameter of $50 \mathrm{~mm}$ has been utilized for the rod model. Distances between the centres of one tip of a core with another are made by $250 \mathrm{~mm}$. Dimensions of work-piece are $1750 \times 750 \times 45 \mathrm{~mm}$. The characteristics of the AK41T material (aluminum alloy) were used in the computations. The material is isotropic and has identical elasticity characteristics under tension and compression, namely, the Young's modulus $\mathrm{E}=7000 \mathrm{~kg} / \mathrm{mm}^{2}$ and Poisson's ratio $\mathrm{v}=0.4$. Steady state creep under both compression and tension in the experiments is described by Norton's law with different coefficients B for each of these types of strain:

- compression: $\mathrm{B}_{1}=0.25 \cdot 10^{-14}\left(\mathrm{~kg} / \mathrm{mm}^{2}\right)^{-\mathrm{n} 1}(\mathrm{~h})^{-1}, \mathrm{n}_{1}=8$; - tension: $\mathrm{B}_{2}=0.5 \cdot 10^{-14}\left(\mathrm{~kg} / \mathrm{mm}^{2}\right)^{-\mathrm{n}^{2}}(\mathrm{~h})^{-1}, \mathrm{n}_{2}=8$.

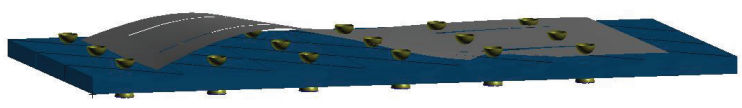

Fig. 1. Finite element model of work-piece and surface with the set curvature

Conditions of contact are modeling slipping without friction.

According to manufacturing process the necessary detail with the set curvature obtain after chipping and milling of previously formed work-piece. 
As initial displacement of contact bodies $\bar{u}_{i}^{0}$ (4) distances from rigid bodies to a surface with required curvature taking into account work-piece thickness are accepted. The residual displacements in an iterative method are determined in nodes of a deformable body nearest to projections of central points of contact bodies to the surfaces of work-piece.

The iterative method is realized in MSC.Marc system by means of a set of user procedures [30].

The numerical model with the set boundary conditions and definitions of projections of contact bodies is implemented by means of user programs in MSC.Patran.

As a result of calculation the anticipatory configuration of work-piece is found (Fig. 2).

In Fig. 3 the work-piece configuration after unloading in comparison with the set surface geometry is shown.

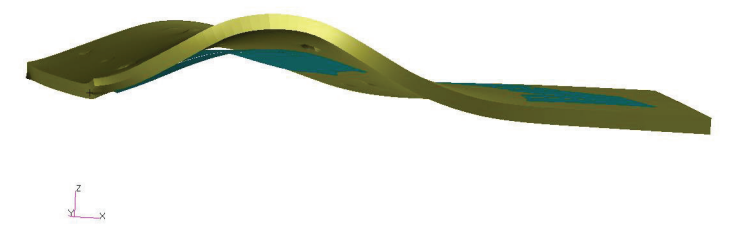

Fig. 2. Anticipatory configuration of work-piece in comparison with the set surface

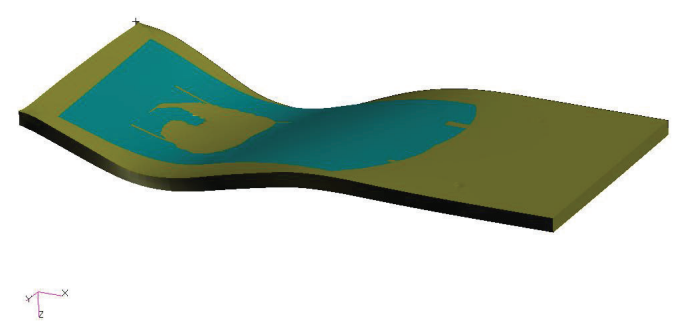

Fig. 3. Residual configration of work-piece in comparison with the set surface

For the iteration method (4), the best convergence coefficients were determined: $\alpha^{k}=2-(1+k)^{-1}$, $\beta^{k}=0.1(1+k)^{-5}$. As a result of solving the inverse problems of shaping with different laws of plates deformation, it is possible to determine the optimal path for stresses and dissipated work (Table 1).

Table 1. Maximum values of stress intensity and dissipated work under different deformation laws.

\begin{tabular}{|c|c|c|c|}
\hline & $\begin{array}{c}\sigma_{i}, \\
\mathrm{~kg} / \mathrm{mm}^{2}\end{array}$ & $A, \mathrm{~kg} / \mathrm{mm}^{2}$ & $e_{k}, \mathrm{~mm}$ \\
\hline$u_{z}=\frac{t}{T} u_{z}^{*}$ & 36.4 & 22 & 28.6 \\
\hline$u_{z}=\sqrt{\frac{t}{T}} u_{z}^{*}$ & 33.3 & 20.9 & 27.8 \\
\hline
\end{tabular}

The convergence of the iterative method in the mean square norm $\left(e_{k}=\left(\sum_{S_{c}}\left(\tilde{u}_{z}{ }^{k}-\tilde{u}_{z}^{0}\right)^{2}\right)^{1 / 2}, S_{c}\right.$ - where is the lower contact surface of the plate and $k$ is the iteration number, $\tilde{u}_{z}^{k}, \tilde{u}_{z}^{0}$ - node parameters describing the residual displacement.

A rational loading path can also be found in the form (3).

\section{Conclusions}

The formulation of inverse quasistatic problems of creep theory in the form of optimal control makes it possible, with the help of the developed algorithm, to find numerical rational solutions for more complex details that are free from representations of the ideal plate and shell, in particular in the formation of wing panels. The developed algorithm is proposed to determine the anticipatory shape (snap-in of a reconfigurable bar device) with the law of change during the deformation process, ensuring minimum damage to the part.

Algorithms developed can be used in industrial applications, such as the forming of aircraft wing panels. It allows simulating and efficiently evaluating the parameters of the parts manufacturing technological processes.

This research was financially supported by the Russian Foundation for Basic Research (16-31-60038 mol_a_dk).

\section{References}

1. V.A.Portnoy, A.V. Portnoy, Vestnik KGTU im. A.N. Tupoleva, 2, 9-13 (2006) [in Russian]

2. V.K. Boresevich, M.V. Zagerniak, V.V. Forgestamp manufacture, 2, 38-41 (2009) [in Russian]

3. B.V. Gorev, I.Zh. Masanov, Technology of mechanical engineering, 7, 13-20 (2009) [in Russian]

4. O.V. Sosnin, B.V. Gorev, I.V. Lyubashevska, Journal of applied mechanics and technical physics, 38, № 2, 293-297 (1997)

5. B.V. Gorev, V.A. Panamarev, V.N. Peretyat'ko, Steel in Translation, 41, №6, 461-463 (2011)

6. B.V. Gorev, V.A. Panamarev, and V.N. Peretyat'ko, Steel in Translation, 41, № 4, 275-277 (2011)

7. O.V. Sosnin, A.F. Nikitenko, B.V. Gorev, Journal of Applied Mechanics and Technical Physics, 51, № 4, 608-614 (2010)

8. I.A. Banshchikova, B.V. Gorev, I.Y. Tsvelodub, Journal of Applied Mechanics and Technical Physics, 48, № 5, 751-754 (2007)

9. I.A. Banshchikova, B.V. Gorev, I.V. Sukhorukov, Journal of Applied Mechanics and Technical Physics, 43, № 3, 448-456 (2002)

10. Y. C. Lin, Y.-Q. Jiang, X.-M. Chen, D.-X. Wen, and H.-M. Zhou, Materials Science and Engineering A, 588, 347-356 (2013) 
11. B.D. Annin, A.I. Oleinikov, K.S. Bormotin, Journal of Applied Mechanics and Technical Physics, 51, № 4, 579-589 (2010).

12. F.C. Ribeiro, E.P. Marinho, D.J. Inforzato, P.R. Costa, G.F. Batalha, Journal of Achievements in Materials and Manufacturing Engineering, 43/1, 353-361 (2010).

13. R. Arabi Jeshvaghani, M. Emami, H.R. Shahverdi, and S. M. M. Hadavi, Materials Science and Engineering A, 528, № 29-30, 8795-8799 (2011).

14. P. R. C. Junior, C. De Moura Neto, and D. A. Wade, Materials Research, 17, № 3, 603-611 (2014).

15. R. Arabi Jeshvaghani, H. Zohdi, H.R. Shahverdi, M. Bozorg, S.M.M. Hadavi, Materials characterization, 73, pp. 8-15 (2012).

16. D. F. Walczyk, J. Lakshmikanthan, and D. R. Kirk, Journal of Manufacturing Systems, 17, № 4, 287296 (1998).

17. D. F. Walczyk and D. E. Hardt, Journal of Manufacturing Systems, 17, № 6, 436-454 (1998).

18. E. Haas, R. C. Schwarz, and J. M. Papazian, Journal of Manufacturing Processes, 4, № 1, 77-85 (2002).

19. D. Simon, L. Kern, J. Wagner, and G. Reinhart, Procedia CIRP, 17, 853-858 (2014).

20. S.Z. Su, M.Z. Li, C.G. Liu, C.Q. Ji,R. Setchi, J. Larkiola, I. Panteleev, I. Stead, R. Lopez, Key Engineering Materials, 504-506, 839-844 (2012).

21. J.J. Chen, M.Z. Li, W. Liu, C.T.Wang, The International Journal of Advanced Manufacturing Technology, 25, 935-939 (2005).

22. S. Y. Hwang, J. H. Lee, Y. S. Yang, and M. J. Yoo, CAD Computer Aided Design, 42, № 11, 10011012 (2010).

23. K.S. Bormotin, Computational mathematics and mathematical physics, 53, №12, 1908-1915 (2013).

24. P. Wriggers, Computational Contact Mechanics ( 2006).

25. Bormotin K.S., Oleinikov A.I., Journal of Applied Mechanics and Technical Physics, 53, №5, 751-760 (2012) .

26. Bormotin K.S., Journal of Applied and Industrial Mathematics, 6, №4, 421-430 (2012).

27. Bormotin K.S., Computational methods and programming, 15, 222-228 (2014) [in Russian].

28. K.-J. Bathe, Finite element procedures (1982).

29. Marc 2016, Vol A: Theory and User Information, MSC.Software Corporation http://www.mscsoftware.com/product/marc.

30. Bormotin K.S., Belykh S.V. and Vin Aung, Computational methods and programming, 17, 258267 (2016) [in Russian]. 